\title{
.
}

\section{Tuberculosis in cattle: the results of the four-area project}

\author{
John M. Griffin, Simon J. More, Tracy A. Clegg, John D. Collins, lan O'Boyle, David H. Williams, Gabrielle E. Kelly, Eamon \\ Costello, D. Paddy Sleeman, Finbarr O'Shea, Murt Duggan, James Murphy and Desmond P.T. Lavin
}

\author{
Centre for Veterinary Epidemiology and Risk Analysis, UCD Veterinary Sciences Centre, UCD and \\ School of Agriculture, Food Science and Veterinary Medicine, College of Life Sciences, University College Dublin, \\ Belfield, Dublin 4, Ireland.
}

The four-area project was undertaken to further assess the impact of badger removal on the control of tuberculosis in cattle herds in Ireland. It was conducted between 1997 and 2002 in matched removal and reference areas in four counties, namely Cork, Donegal, Kilkenny and Monaghan, representing a wide range of Irish farming environments. In the removal areas, a proactive programme of badger removal was conducted, on two or three occasions each year, whereas in the reference areas, badger removal was entirely reactive following severe outbreaks of tuberculosis amongst cattle. A detailed statistical analysis of this study has already been presented by Griffin et al. (2005); this paper presents further, mainly descriptive, findings from the study. In total, 2,360 badgers were captured in the removal areas of which 450 (19.5\%) were considered positive for tuberculosis and 258 badgers were captured in the reference areas, with 57 (26.1\%) positive for tuberculosis. The annual incidence of confirmed herd restrictions was lower in the removal area compared to the reference area in every year of the study period in each of the four counties. These empirical findings were consistent with the hazard ratios found by Griffin et al. (2005). Further, the effect of proactive badger removal on cattle tuberculosis in the four-area project and in the earlier east-Offaly project, as measured using the number of reactors per I,000 cattle tested, were very similar, providing compelling evidence of the role of badgers in the epidemiology of tuberculosis in Irish cattle herds. The validity of the four-area project was discussed in detail. Efforts to minimise badger-to-cattle transmission in Ireland must be undertaken in association with the current comprehensive control programme, which has effectively minimised opportunities for cattle-to-cattle transmission.

\begin{abstract}
Introduction
Wildlife are now recognised as an important reservoir of bovine tuberculosis in a number of countries, including New Zealand, the United States, Canada and South Africa (de Lisle, 2002). There has been building evidence of the role of infected badgers (Meles meles, a protected species in Ireland) as a reservoir of Mycobacterium bovis in Ireland and the UK, including: isolation of $M$. bovis in badgers in several countries (Noonan et al., 1975; Bouvier et al., 1962 cited by Cheeseman et al., 1989; Krebs, 1997); recognition that badgers were highly susceptible to M. bovis infection (Gormley and Costello, 2003), and that tuberculosis was endemic within the badger population in Ireland (O'Boyle et al., 2003); and the identification of identical strains of M. bovis in local Irish cattle and badger populations (Costello et al., 1999). However, this information on its own is not sufficient to prove disease causation. Further, these findings do not provide quantitative

\section{Corresponding author:}

Simon J. More

Centre for Veterinary Epidemiology and Risk Analysis, UCD Veterinary Sciences Centre, UCD School of Agriculture, Food Science and Veterinary Medicine, College of Life Sciences, University College Dublin, Belfield, Dublin 4, Ireland.

E-mail:simon.more@ucd.ie
\end{abstract}

evidence of the importance of badger-cattle transmission of $M$. bovis in the epidemiology of bovine tuberculosis in Ireland.

In such circumstances, a field trial offers the best opportunity to critically assess the impact of badger removal on the control of tuberculosis in cattle herds in Ireland, thereby determining the relationship - in terms of $M$. bovis infection - between badgers and cattle. These objectives have now been evaluated in the east-Offaly project (conducted during 1989 to 1995) and the four-area project (1997-2002). The east-Offaly project provided robust evidence of the link between tuberculous badgers and tuberculosis in cattle (Eves, 1999). In this project, badger removal resulted in a statistically significant decrease in the risk of disclosure of a tuberculin reactor in a herd, with the odds of such a disclosure being 14 times greater in

\section{Glossary:}

Reference areas: study areas where disturbance of badger populations was minimised; badger capture was only conducted following severe outbreaks of tuberculosis in cattle herds.

Removal areas: study areas where a proactive programme of badger removal was carried out on two to three occasions each year during the study.

CRR (Confirmed restriction risk): the number of new confirmed herd restrictions per 100 herds per annum.

APT: the number of tuberculin reactors per thousand animal tests. 
Table I: Numbers of badgers captured in the treatment areas, by year and county, prior to the start of the study and during the study Area Period Year Cork Donegal Kilkenny Monaghan Total

\begin{tabular}{|c|c|c|c|c|c|c|}
\hline Removal areas & $1988-92$ & 31 & 118 & 0 & 3 & 152 \\
\hline Pre-study period ${ }^{a}$ & $1992 / 93$ & II & 0 & 0 & 0 & II \\
\hline & 1993/94 & 9 & 0 & 0 & 34 & 43 \\
\hline & $1994 / 95$ & 0 & 5 & 4 & 56 & 65 \\
\hline & $1995 / 96$ & 0 & 4 & 148 & 123 & 275 \\
\hline & 1996/97 & 1 & 0 & 57 & 0 & 58 \\
\hline & Total & 21 & 9 & 209 & 213 & 452 \\
\hline Study period & $1997 / 98$ & 235 & 191 & 189 & 176 & 791 \\
\hline & 1998/99 & 103 & 38 & 83 & 84 & 308 \\
\hline & 1999/00 & 46 & 16 & 61 & 71 & 194 \\
\hline & $2000 / 0 I^{b}$ & 29 & 16 & 28 & 34 & 107 \\
\hline & $2001 / 02$ & 36 & 16 & 49 & 78 & 179 \\
\hline & Total & 449 & 277 & 410 & 443 & 1,579 \\
\hline Buffer areas & $1988-92$ & 16 & 0 & 0 & 0 & 16 \\
\hline Pre-study period & $1992 / 93$ & 0 & 0 & 0 & 0 & 0 \\
\hline & $1993 / 94$ & 0 & 0 & 0 & 7 & 7 \\
\hline & $1994 / 95$ & 0 & 0 & 0 & 3 & 3 \\
\hline & $1995 / 96$ & 2 & 0 & 0 & I & 3 \\
\hline & $1996 / 97$ & 0 & 0 & 0 & 0 & 0 \\
\hline & Total & 2 & 0 & 0 & I I & 13 \\
\hline Study period & $1997 / 98$ & 166 & 17 & 61 & 78 & 322 \\
\hline & 1998/99 & 85 & 16 & 16 & 42 & 159 \\
\hline & $1999 / 00$ & 53 & 10 & 14 & 34 & III \\
\hline & $2000 / 0 I^{b}$ & 22 & 6 & 18 & 20 & 66 \\
\hline & $2001 / 02$ & 31 & 16 & 33 & 43 & 123 \\
\hline & Total & 357 & 65 & 142 & 217 & 781 \\
\hline Reference areas & $1988-92$ & 0 & 0 & 0 & 5 & 5 \\
\hline Pre-study period ${ }^{a}$ & $1992 / 93$ & 0 & 0 & 0 & 0 & 0 \\
\hline & $1993 / 94$ & 5 & 0 & 0 & 17 & 22 \\
\hline & $1994 / 95$ & 4 & I & 55 & 0 & 60 \\
\hline & $1995 / 96$ & 5 & I & 33 & 0 & 39 \\
\hline & $1996 / 97$ & 3 & 4 & 4 & 0 & II \\
\hline & Total & 17 & 6 & 92 & 17 & 132 \\
\hline Study period & $1997 / 98$ & 18 & 0 & 7 & 4 & 29 \\
\hline & 1998/99 & 36 & 9 & 43 & 21 & 109 \\
\hline & $1999 / 00$ & 23 & 0 & 3 & 17 & 43 \\
\hline & $2000 / 0 I^{b}$ & 14 & 4 & 16 & 10 & 44 \\
\hline & $2001 / 02$ & 6 & 0 & 25 & 2 & 33 \\
\hline & Total & 97 & 13 & 94 & 54 & 258 \\
\hline
\end{tabular}

a The pre-study period: September I, 1992 to August 31, 1997

${ }^{b}$ The number of badgers removed in $2000 / 0$ I was low due to restrictions imposed following the outbreak of foot-and-mouth disease in Ireland.

the control area (i.e., reference) than in the project area (i.e., removal) in the final year of the study (Ó Máirtín et al., 1998). The four-area project has built on this earlier work. It was conducted in four different counties to represent a wide range of farming environments. Further, study areas were chosen to minimise the opportunity for badger migration into designated removal areas. A detailed statistical analysis of this study has been presented by Griffin et al. (2005). In agreement with the findings of the east-Offaly project, both the odds and hazard ratios of a confirmed restriction in the removal areas during the five-year study period were significantly lower than in the matched reference areas. This paper presents further, mainly descriptive, findings from the four-area project.

\section{Materials and methods}

The study was conducted between September I, 1997 and August 31, 2002 in matched removal and reference areas in counties Cork, Donegal, Kilkenny and Monaghan. Selection criteria for the removal areas included higher-than-average apparent disease prevalence, natural geographical boundaries, where possible, to prevent badger migration, and areas considered representative of the diverse Irish landscape. Where natural barriers were absent, 'buffer areas' were created, up to $6 \mathrm{~km}$ in width, at the boundary of each selected removal area. A range of matching criteria were used during the selection of each reference area, based on factors known to influence badger density and herd prevalence of tuberculosis, including county, livestock density, herd size, farm-enterprise type, disease prevalence (measured 
Table 2:Average annual badger removal intensity, in the treatment areas, during the pre-study period and the study period, by year and county (removals per $\mathrm{km}^{2}$ per year)

\begin{tabular}{lrrrrr} 
Year & Cork & Donegal & Kilkenny & Monaghan & Total \\
$\begin{array}{l}\text { The pre-study period } \\
\text { Removal areas }\end{array}$ & 0.02 & 0.01 & 0.17 & 0.14 & 0.09 \\
\hline $\begin{array}{l}\text { Buffer areas } \\
\text { Reference areas }\end{array}$ & 0.003 & 0.00 & 0.00 & 0.03 & 0.01 \\
\hline $\begin{array}{l}\text { The study period } \\
\text { Removal areas }\end{array}$ & 0.02 & 0.004 & 0.07 & 0.01 & 0.03 \\
\hline $\begin{array}{l}\text { Buffer areas } \\
\text { Reference areas }\end{array}$ & 0.48 & 0.60 & & & 0.29 \\
\hline
\end{tabular}

using APT) during the nine years prior to the study, and selected geographic features. Further details of the selection criteria can be found in Griffin et al. (2005). The matched removal and reference areas in each county were supervised by a single District Veterinary Office (DVO), and managed by a single, appropriately trained, team throughout the study period.

A comprehensive survey of badger habitat and activity, to locate and describe all setts, was carried out in the removal, buffer and reference areas. Licences for the removal of badgers for scientific purposes were issued by the National Parks and Wildlife Section of Dúchas, the Irish Heritage Service. Further details of the badger capture procedures, are given in the Badger Manual prepared by the Department of Agriculture, Food and Forestry (DAFF, 1996).

In the removal and buffer areas, a proactive programme of badger removal was conducted, with the aim to achieve and sustain a high level of badger removal throughout the study period. Badger removal was conducted under licence on two or three occasions each year between September I, 1997 and August 31, 2002. In each of the reference areas, badger removal was entirely reactive. It was only conducted on a holding and its surrounds provided there were four or more standard tuberculin reactors at an outbreak of bovine tuberculosis, and the investigating Veterinary Inspector, after taking other factors into account, considered that tuberculous badgers were likely to be the source of the outbreak. A gross post-mortem examination was conducted on all badgers. If evidence of tuberculosis was not found, a pool of designated tissues (bronchial and mediastinal lymph nodes, retropharyngeal lymph nodes, a portion of each kidney and a portion of lung tissue) was sent for histopathological examination and culture. A badger was regarded as positive for tuberculosis if it was positive at histopathological examination and/or on culture.

Only herds that were totally contained within either the removal areas or the reference areas were included in the analyses. The national tuberculin testing programme was conducted, without modification, in the study areas throughout the study period. The interferon- $\gamma$ test was used, as described previously (Monaghan et al., 1994), at the discretion of the investigating veterinarian to identify residually-infected animals in breakdown herds. For the purposes of this study, a confirmed tuberculosis herd restriction was defined as a herd restriction at which a tuberculous lesion was recorded in one or more animals at post-mortem examination at the time or during the course of the restriction. A major confirmed tuberculosis herd restriction, a subset of the above-mentioned, was defined as a herd restriction at which a tuberculous lesion was recorded in one or more animals at post-mortem examination, and at least two standard reactors were recorded at a tuberculin test in the course of the restriction.

The incidence of confirmed herd restrictions was measured using confirmed restriction risk (CRR). If a herd had a confirmed restriction more than once in a year, it was only counted once in the numerator. During this study, a year was defined as commencing on Ist September and ending on August $3 \mathrm{I}$ in the following year. The CRR and the severity of restrictions were examined by time, county and treatment area. Data from before the start of the study were included in the analysis in order to permit a comparison of tuberculosis levels in the reference and removal areas prior to the study. Further, an average CRR was calculated for three time periods: the 'pre-study' period (from September I, 1992 to August 3I, 1997, inclusive) and two periods in the 'study' period (from September I, 1997 to August 3I, 2000, and from September I, 2000 to August 3I, 2002, inclusive). The APT was calculated as described previously (Collins and Hammond, 2003).

\section{Results}

The total size of the removal and reference areas (excluding the buffer areas) was $1,961 \mathrm{~km}^{2}$, i.e., approximately $3.9 \%$ of the agricultural land area of the Republic of Ireland. There was a high level of cooperation and support from the landowners in each area. Permission to survey was refused on only one occasion, on a single holding of 19 hectares in County Cork.

Of the 2,360 badgers captured in the removal and buffer areas during the study, I, I I 3 (47.2\%) were caught during the first year of removal (Table I). In the reference areas, 258 badgers were removed during the study. During the five-year pre-study period, the average annual removal intensity in the removal areas $\left(0.09\right.$ badgers removed per $\mathrm{km}^{2}$ per year) and in the reference areas $(0.03)$ was broadly similar to the intensity of badger removal in the reference areas during the study period (0.05). This was substantially less than the intensity of badger removal in the removal areas (0.33) during the study (Table 2).

A full post-mortem examination was conducted on 2,3I0 (97.9\%) of the badgers captured in the removal and buffer areas during the study period, of which 450 (19.5\%) were considered positive for tuberculosis. Of the 258 badgers captured in the reference areas during the study period, $218(84.5 \%)$ underwent a full postmortem examination. Of these, 57 (26.1\%) were deemed positive for tuberculosis. 

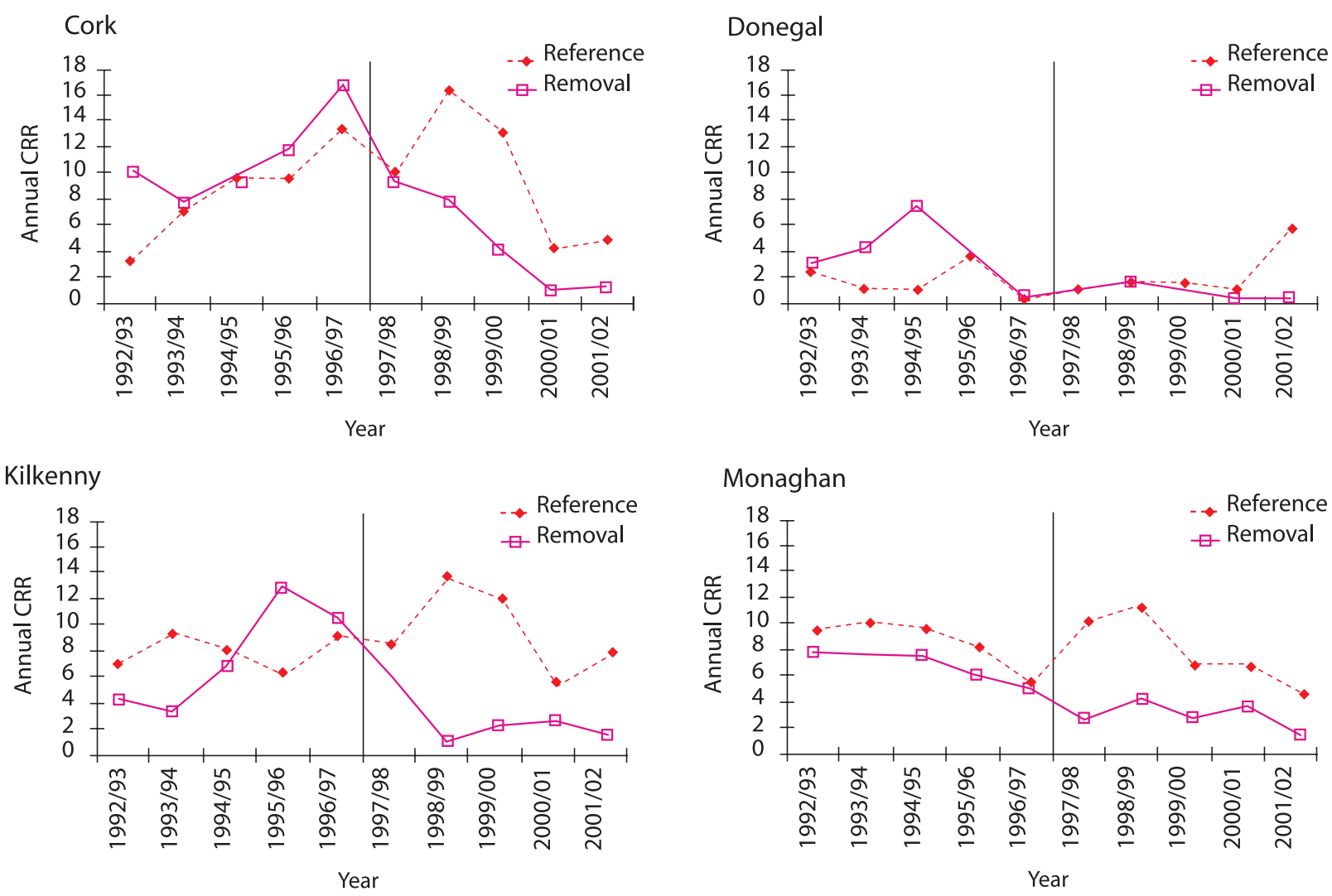

Figure 2:Annual confirmed restriction risk (CRR) over time and by county, in the removal areas and reference areas.

during the pre-study and study periods are presented in Table 3. In each county and with respect to confirmed herd restrictions, the number increased in each of the reference areas and decreased in each of the removal areas during the study period as compared to the pre-study period. The pattern of unconfirmed herd restrictions was less consistent.

APT had been used previously to measure tuberculosis levels in the east-Offaly project (Eves, 1999). The APT in the project area of the east-Offaly project and in the removal areas of the four-area projects are shown in Figure 3. Although the studies were conducted in different areas under different time periods, the pattern of change in APT following badger removal is remarkably similar.

\section{Discussion}

It is critical that this study is considered within a clearly-defined context. The Irish control programme is comprehensive, incorporating each of the accepted elements of disease control, including mandatory annual tuberculin testing of all animals in the national herd and early, ongoing removal of infected animals. As a direct consequence of these efforts, cattle-to-cattle transmission has become relatively less important in the epidemiology of bovine tuberculosis in Ireland. It has long been suspected that another source of the bovine tuberculosis bacillus is involved, given the lack of national progress towards eradication despite these efforts. These suspicions have now been confirmed, with the results from the four-area project (and the earlier east-Offaly project; Figure 3) clearly highlighting wildlife (and, specifically, transmission of $M$. bovis from badgers to cattle) as a key constraint to disease eradication in Ireland. These findings are of national importance and provide compelling evidence of the linkage between proactive badger removal and a reduction in the level of tuberculosis in Irish cattle in the areas concerned. Note that this linkage (and the consequent impact of proactive badger removal) would not have been as evident if the national control programme were less effective; to illustrate, if cattle-to-cattle transmission were still common, differences in disease incidence between the removal and reference areas would not have been as marked.

In each of the four areas, there were differences between the removal area and reference area in terms of the number of confirmed tuberculosis breakdowns in cattle during the study period. The CRR was lower in every year of the study period in each removal area as compared to the corresponding reference area (Figures $\mathbf{I}$ and 2). In addition, the herd incidences of tuberculosis in each removal area were considerably lower in the study period than during the pre-study period. Griffin et al. (2005) identified significant differences between the removal area and reference area in each of the four counties during the study period, both in the probability of, and the time to, a confirmed herd restriction due to tuberculosis; to illustrate, in the final year of the study the odds of a confirmed herd restriction in the removal areas as compared to the reference areas was 0.25 in Cork, 0.04 in Donegal, 0.26 in Kilkenny and 0.43 in Monaghan. Thus, the odds of a confirmed restriction in the removal area compared to the reference area ranged from 2.3 times less likely (Monaghan) to 25 times less likely (Donegal). Furthermore, the hazard ratios (removal over reference) ranged from 0.4 to 0.04 ; that is, a $60 \%$ to $96 \%$ decrease in the rate at which herds became the subject of a confirmed restriction. Although there was a general decrease in CRR in each of the reference areas, except Donegal, during the study period (Figure 2), this effect was not significant (Griffin et al., 2005). A decrease in the 


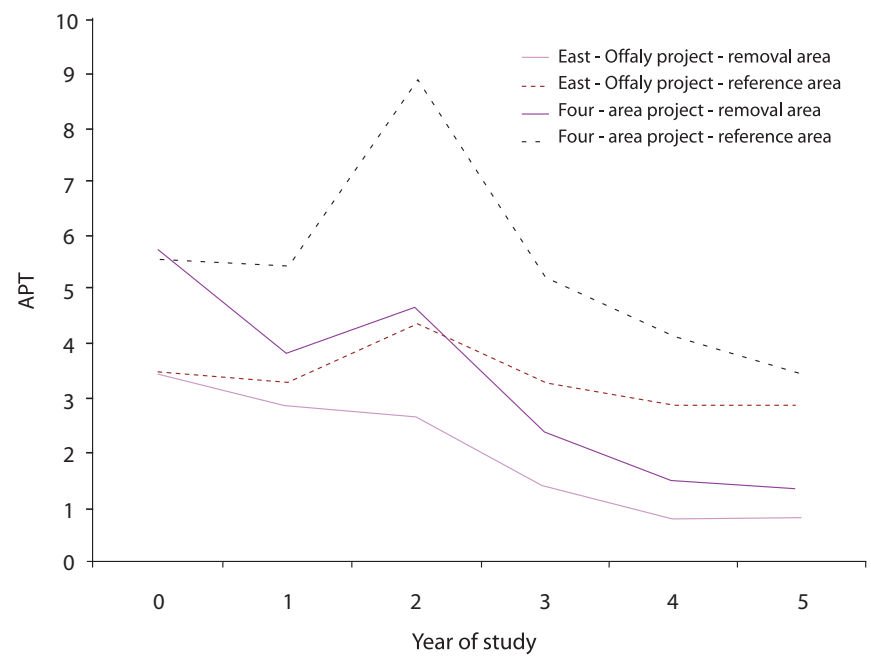

Figure 3: Comparison of the numbers of tuberculin reactors per 1,000 animal tests (APT) in the east-Offaly removal ('project') and reference ('control') areas, and in the combined four-area removal and reference areas, during the first five years of each study.

national CRR was also observed during this period, although perhaps to a lesser extent. With the use of a contemporaneous control, it has been possible to distinguish treatment from unrelated effects.

The CRR was used in this paper as the preferred measure of disease incidence. This is at odds with the widespread field use of APT in Ireland, which provides a measure of the reactor disclosure rate per I,000 animals tested. However, there is a range of difficulties with the APT, including the influence of testing intensity on this measure (as the intensity of testing increases, the denominator is likely to increase more quickly than the numerator), the inclusion of results from both surveillance and control activities, and the focus on the animal rather than on the herd. Because Irish herds are tested at least yearly, the CRR is an appropriate herd-level measure of incidence, focusing on the number of new herd restriction confirmations during each I2-month period. It is important to note that measures of incidence and of prevalence were both used in the detailed analysis of this study (Griffin et al., 2005), during survival and logistic modelling, respectively, for reasons previously discussed. The results from these differing analyses were remarkably similar.

There appears to be a relationship between breakdown severity and badger removal (Table 3 ). When the pre-study and study periods were compared across all four counties, the reduction in the number of major confirmed restrictions was consistently greater than the reduction in all confirmed restrictions. For example, in the Kilkenny removal area, these reductions were $82 \%$ and $59 \%$, respectively. This finding is consistent with anecdotal field evidence, and previously had been noted with respect to the east-Offaly project (Eves, 1999). However, these are descriptive results and do not take account of potential confounding by other variables, such as herd size or previous history of the disease within the herd; consequently, they should be interpreted with care.

We have conducted a detailed evaluation of the internal validity of this study (that is, whether the study results accurately reflect reality within the study population). A summary of key findings is presented in the following paragraphs, and further detail is available elsewhere (Centre for Veterinary Epidemiology and Risk Analysis, 2004; Griffin et al., 2005; More, 2005).
In this study, strategic badger removal was conducted in the reference areas following severe outbreaks of disease in local cattle herds. This approach, which was consistent with official government policy throughout the rest of Ireland, was critical to the high levels of cooperation achieved throughout the study period. Further, the criteria for badger removal were considerably more stringent in the reference areas than throughout the rest of Ireland, thereby limiting badger disturbance in these areas. A national programme of strategic badger removal was not uniformly established throughout Ireland until towards the end of the 1990s (that is, approximately from year one of the study). Consequently, the measured increase in the intensity of badger removal in the reference areas between the pre-study period ( 0.03 badgers removed $/ \mathrm{km}^{2} /$ year) and study period $(0.05)$ (Table 2 ) is not at odds with the above-mentioned criteria, rather it is a reflection of a national programme of strategic badger removal at an early stage of development. Concerns have been expressed about the use of reactive badger removal in the reference areas; most notably by Donnelly et al. (2003) who suggested that true treatment effect may have been overestimated due to unanticipated effects from this strategy. As indicated previously, there is no evidence to support these concerns (Griffin et al., 2005), based on a comparison of CRR in the reference areas prior to and during the study period, after considering the intensity of badger removal in the reference areas throughout the study period, and after reviewing the temporal relationship between badger removal and herd breakdowns in each of the reference areas. In addition, although there was an observed increase in CRR in each of the reference areas (except Donegal) during the second year of the study (1998/99; Figure 2), a similar national CRR increase was also observed at this time.

There are several important issues relating to the selection of the matched reference and removal areas in each county. As discussed previously and elsewhere (Griffin et al., 2005), the removal and reference areas were chosen purposively, using selection and matching criteria. Area security (that is, areas with minimal opportunity for ongoing migration of badgers) was used as a key criterion during removal area selection, partly in response to concerns from the earlier east-Offaly project (Philips et al., 2003), where the dough-nut design facilitated the inward migration of badgers from the control (equivalent, in the four-area project, to the reference area) to the project (removal) area. In hindsight, this issue was of limited concern, noting that any inward migration of badgers from the control to the project area in the east-Offaly project will have had the effect of making it more difficult to detect a treatment effect, if one was present. Because the matched areas were not selected at random, it is important to critically evaluate the equivalence of these areas at study start, in terms of factors that might reasonably influence CRR throughout the study period. In the four-area project, the matched areas were broadly similar according to a range of attributes, relating to cattle numbers, average herd size, grazing density, and farm type (Griffin et al., 2005). However, there were three important differences between the matched areas which should be highlighted. Firstly, there were topographical differences between the matched areas of Kilkenny and Monaghan (that is, areas higher than $200 \mathrm{~m}$ were present in the removal areas but not the reference areas of Kilkenny and Monaghan; see Figure 2 in Griffin et al., 2005). Consequently, there were some differences in the spatial pattern of land use; in 
particular, coniferous forest, peat bogs, low productivity grassland (Kilkenny only) and land principally occupied by agriculture with significant areas of natural vegetation were more common in the removal areas in these counties (Hammond, 1996). As a result of this difference, but also because upland is negatively associated with badger density in Ireland (Smal, 1995), it is conceivable that there was less opportunity for badger-to-cattle interaction in the removal as compared with the reference areas of Kilkenny and Monaghan. Logically, this will have made it more difficult to detect a treatment effect, if present. Secondly, during the two years prior to study start, a substantial number of badgers were removed from the removal areas of Kilkenny and Monaghan and, to a lesser extent, the reference area of Kilkenny (Table I). Although there was no immediate impact on CRR (consistent with biological plausibility and later study findings), these operations could reasonably explain the earlier appearance (as presented in Table 6, Griffin et al., 2005) of a significant difference in the odds of a confirmed herd restriction between the removal area and the reference area in Monaghan (observed from year one of the study onwards) compared with the areas in Cork, Kilkenny (both observed from year two onwards) and Donegal (observed only in year five). Thirdly, in all counties but Monaghan, there were significant differences in the odds of a confirmed herd restriction between matched areas in at least one of the years prior to study-start; however, in each case the odds were greater in the removal area than in the reference area. Therefore, any residual difference between the reference and removal areas at the time of study-start will also have had the effect of reducing the probability of detecting any treatment effect during the study, if present (Griffin et al., 2005). In conclusion, although differences were observed both prior to (residual differences in the odds of a confirmed herd restriction at study start, differences in the intensity of badger removal during the pre-study period) and during the study (differences in land use), the treatment effect was remarkably consistent in all counties (Table 2 ) and similar to those observed previously in the east-Offaly project.

Throughout the study period, care was taken to minimise the potential for measurement bias. In each county, the matched reference and removal areas were supervised by the same field people within the same administrative unit. Further, the project was coordinated nationally, and all work (both field and laboratory) was conducted in accordance with nationally-agreed protocols (Griffin et al., 2005). There was minimal use of the interferon- $\gamma$ test in the study areas, and no evidence of variation - between removal and reference areas - in the intensity of removal of residually-infected animals from breakdown herds. Although as yet unquantified, the level of animal movement within matched areas was likely to be very similar. The field testing was conducted by a range of private veterinarians, and factory surveillance by a range of factories; however, we are not aware of any systematic differences in the quality of field testing or factory surveillance in matched areas.

The observed badger density, as estimated from the intensity of badger removal during the first year of the study, varied between counties. Some variation is inevitable, given county differences in terms of habitat composition. For example, pasture accounts for $89 \%$ of the total land area in Cork, $73 \%$ in Monaghan, $71 \%$ in Kilkenny and $37 \%$ in Donegal (Hammond, 1997). However, other factors are clearly important, given the intensity of badger removal in the
Donegal removal area in the first year of the study period $\left(0.89 / \mathrm{km}^{2}\right)$, compared with 0.75 and 0.58 for Kilkenny and Monaghan, respectively. This variation might be explained, in part, by the effect of removal history on badger density. In Kilkenny and Monaghan, there were official programmes of badger removal during the three to four years immediately prior to study start. In contrast, there was very little badger capture after August 1992 in Donegal (Table I). The varying badger capture rates may also reflect varying levels of boundary security in the different counties.

In this study, the prevalence of tuberculosis in badgers $(19.4 \%$ in the removal areas and $26.1 \%$ in the reference areas) was considerably higher than estimates from a range of previous Irish studies, including routine post-mortem examination of animals removed under license with an annual prevalence ranging from $12.2 \%$ to $13.3 \%$ (O'Boyle, $1999,2000,2002)$ and $11.6 \%$ prevalence found during a study of road casualty accidents (O'Boyle et al., 2003). This increase is likely a reflection of improved diagnostic methods rather than any change in actual disease prevalence (Costello et al., 1998). Further information about tuberculosis in badgers captured during the four-area project will be available shortly.

Based on the results of this study, an effective scheme to control tuberculosis in badgers, with which cattle may come in contact, is now recognised as a prerequisite for the eradication of tuberculosis from the Irish cattle population (Gormley and Collins, 2000). This presents significant challenges for scientists and policy-makers (Gormley and Costello, 2003) for a number of reasons. These include the international legal protection, and national status, afforded to badgers, and the increasing number of badgers nationally as a consequence of agricultural intensification and increase in productive pastures. Further reasons include the close physical proximity of badgers and cattle, given the preference of badgers in Ireland to locate setts in hedgerows (Hammond et al., 200I) and the high prevalence of disease among the Irish badger population (L. Corner, personal communication). Ireland is currently implementing a comprehensive strategy to address these challenges. In the short-term, the Department of Agriculture and Food is implementing a national programme for wildlife control, with greatest focus in areas of high disease prevalence, when and where wildlife are implicated in on-farm breakdowns of bovine tuberculosis (O'Keeffe et al., 2002). In these areas, along with other measures, strategic badger removal will form the basis of temporary disease control (by minimising contact between cattle and infected badgers), and will also provide potential locations for vaccination trials and later usage (O'Keeffe et al., 2002). In the longer term, Ireland is committed to the development and application of an effective badger vaccine, with the aim to reduce $M$. bovis transmission between infected badgers and susceptible cattle (Gormley and Costello, 2003). The feasibility of such an approach was first considered in 1994, with inputs from scientists from both the Republic of Ireland and Northern Ireland (Ellis et al., 1994). Current work is focusing on the use of a live vaccine based on $M$. bovis BCG which may persist in the host and continuously prime the protective cellular immune response (Gormley and Costello, 2003). Results from experimental studies have been promising.

\section{Acknowledgements}

The authors acknowledge the contribution of staff of the 
Mycobacteriology Section, Central Veterinary Research Laboratory, Abbotstown and staff at Regional Veterinary Laboratories, District Veterinary Offices and The Irish Equine Centre.

\section{References}

Centre for Veterinary Epidemiology and Risk Analysis (2004). The Impact of Badger Removal on the Control of Tuberculosis in Cattle Herds in Ireland. A report to the Minister of Agriculture and Food, from University College Dublin, Dublin.

Cheeseman, C.L., Wilesmith, J.W. and Stuart, F.A. (I989). Tuberculosis: the disease and its epidemiology in the badger, a review. Epidemiology and Infection I03: I 13-125.

Collins, J.D. and Hammond, R.F. (2003). Summary statistics 2002, In: Selected papers 2002-2003. Veterinary Epidemiology and Tuberculosis Investigation Unit, University College Dublin, Dublin, PP 73-87.

Costello, E., O'Grady, D., Flynn, O., O'Brien, R., Rogers, M., Quigley, F., Egan, J. and Griffin, J. (1999). Study of restriction fragment length polymorphism analysis and spoligotyping for epidemiological investigation of Mycobacterium bovis infection. Journal of Clinical Microbiology 37: 3217-3222.

Costello, E., Quigley, F., O'Grady, D., Flynn, O., Gogarty, A., McGuirk, J., O'Rourke, J. and Griffin, J. (1998). Laboratory examination of tissues from badgers and cattle from the fourarea study for evidence of tuberculosis, In: Selected Papers 1997. Tuberculosis Investigation Unit, University College Dublin, Dublin, PP 32-36.

DAFF (1996). Department of Agriculture Food and Forestry, Badger Manual. Dublin, Ireland: DAFF.

de Lisle, G.W., Bengis, R.G., Schmitt, S.M., and O'Brien, D.J. (2002). Tuberculosis in free-ranging wildlife: detection, diagnosis and management. Revue Scientifique et Technique Office International des Epizooties 21: 317-334.

Donnelly, C.A., Woodroffe, R., Cox, D.R., Bourne, J., Gettinby, G., Le Fevre, A.M., McInerney, J.P. and Morrison, W.I. (2003). Impact of localized badger culling on tuberculosis incidence in British cattle. Nature 426: 834-837.

Ellis, W., Collins, J.D., Feore, M., Neill, S., Sheridan, M., Sleeman, D., Hughes, S. and Rogers, M. (1994). The feasibility of developing a vaccine against tuberculosis for use in the badger (Meles meles). (Department of Agriculture, Food and Forestry, Republic of Ireland and Department of Agriculture for Northern Ireland), PP 52

Eves, J.A. (1999). Impact of badger removal on bovine tuberculosis in east County Offaly. Irish Veterinary Journal 52: 199-203.

Gormley, E. and Collins, J.D. (2000). The development of wildlife control strategies for eradication of tuberculosis in cattle in Ireland. Tubercle and Lung Diseases 80: 229-236.

Gormley, E. and Costello, E. (2003). Tuberculosis and badgers: new approaches to diagnosis and control. Journal of Applied Microbiology 94: 80s-86s.

Griffin, J.M., Williams, D.H., Kelly, G.E., Clegg, T.A., O'Boyle, I., Collins, J.D. and More, S.J. (2005). The impact of badger removal on the control of tuberculosis in cattle herds in Ireland. Preventive Veterinary Medicine 67: 237-266.

Hammond, R.F. (1997). Variables used to characterise the four areas badger survey removal/reference areas. In: Selected Papers
1996. Veterinary Epidemiology and Tuberculosis Investigation Unit, University College Dublin, Dublin, PP I2-I8.

Hammond, R.F., McGrath, G. and Martin, S.W. (200I). Irish soil and land-use classifications as predictors of number of badgers and badger setts. Preventive Veterinary Medicine 5 I: I37- 48.

Krebs, J.R. (1997). Bovine Tuberculosis in Cattle and Badgers. London, UK: Ministry of Agriculture, Fisheries and Food, p 191.

Monaghan, M., Doherty, M.L., Collins, J.D., Kazda, J.F. and Quinn, P.J. (1994). The tuberculin test. Veterinary Microbiology 40: I79191.

More, S.J. (2005). Towards eradication of bovine tuberculosis in Ireland: a critical review of progress. In: Proceedings, 2005 Annual Meeting, Society for Veterinary Epidemiology and Preventive Medicine. Nairn, Scotland. Edited by D. J. Mellor, A. M. Russell and J. L. N. Wood. PP 13-23.

Noonan, N.L., Sheane, W.D., Harper, L.R. and Ryan, P. J. (1975). Wildlife as a possible reservoir of bovine TB. Irish Veterinary Journal 29: I.

Ó Máirtín, D., Williams, D.H., Griffin, J.M., Dolan, L. and Eves, J.A. (1998). The effect of a badger removal programme on the incidence of tuberculosis in an Irish cattle population. Preventive Veterinary Medicine 34: 47-56.

O'Boyle, I. (1999). Review of badger (Meles meles) research licences in 1998, In: Selected Papers 1998. Tuberculosis Investigation Unit, University College Dublin, Dublin, Pp 10-I4.

O'Boyle, I. (2000). Review of badger (Meles meles) research licences in 1999, In: Selected Papers 1999. Tuberculosis Investigation Unit, University College Dublin, Dublin, pp I5-2I.

O'Boyle, I. (2002). Review of badger (Meles meles) research licences in 2000 and 200I, In: Selected Papers 2000-200I. Veterinary Epidemiology and Tuberculosis Investigation Unit, University College Dublin, Dublin, pp 19-25.

O'Boyle, I., Costello, E., Power, E.P., Kelleher, P.F., Bradley, J., Redahan, E., Quigley, F., Fogarty, U. and Higgins, I. (2003). Review of badger (Meles meles) research licences in 2002, In: Selected Papers 2002-2003. Veterinary Epidemiology and Tuberculosis Investigation Unit, University College Dublin, Dublin, PP I3-I8.

O'Keeffe, J.J., Hammond, R.F. and McGrath, G. (2002). Density maps highlight areas with chronic bovine tuberculosis and enable targeting of resources to eradicate disease, In: Selected Papers 20002001. Veterinary Epidemiology and Tuberculosis Investigation Unit, University College Dublin, Dublin, PP 4I-44.

Philips, C.J.C., Foster, C.R.W., Morris, P.A. and Teverson, R. (2003). The transmission of Mycobacterium bovis infection to cattle. Research in Veterinary Science 74: I- I5.

Smal, C. (1995). The Badger and Habitat Survey of Ireland. Dublin: Stationary Office. 